\title{
Boron Vacancies Causing Breakdown in 2D Layered Hexagonal Boron Nitride Dielectrics
}

\author{
A. Ranjan ${ }^{1,2,3, \#, \text { N. Raghavan }}{ }^{1}$, F.M. Puglisi ${ }^{4}$, S. Mei ${ }^{1}$, A. Padovani ${ }^{5}$, L. Larcher $^{5}$, \\ K. Shubhakar' ${ }^{1}$, P. Pavan $^{5}$, M. Bosman ${ }^{2,6}$, X.X. Zhang ${ }^{3}$, S.J. O'Shea ${ }^{2}$ and K.L. Pey ${ }^{1}$ \\ ${ }^{1}$ Singapore University of Technology and Design, 8 Somapah Road, 487372, Singapore. \\ ${ }^{2}$ Institute for Materials Research and Engineering, A*STAR, 2 Fusionopolis Way, 138634, Singapore. \\ ${ }^{3}$ King Abdullah University of Science and Technology, Thuwal, 23955, Saudi Arabia. \\ ${ }^{4}$ DIEF-Università di Modena e Reggio Emilia, Via P. Vivarelli 10/1, Modena, 42125, Italy. \\ ${ }^{5}$ Applied Materials, Santa Clara, California, 95054, USA. \\ ${ }^{6}$ National University of Singapore, 9 Engineering Drive 1, 117575, Singapore. \\ "Email: alok_ranjan@mymail.sutd.edu.sg; Phone: +65 63036617
}

\begin{abstract}
Dielectric breakdown in 2-D insulating films for future logic device technology is not well understood yet, in contrast to the extensive insight we have in the breakdown of bulk dielectric films such as $\mathrm{HfO}_{2}$ and $\mathrm{SiO}_{2}$. In this study, we investigate the stochastic nature of breakdown (BD) in hexagonal boron nitride (h-BN) films using ramp voltage stress and examine the BD trends as a function of stress polarity, area and temperature. We present evidence that points to a non-Weibull distribution for h-BN BD and use multi-scale physics-based simulations to extract the energetics of the defects that are precursors to $\mathrm{BD}$, which happens to be boron vacancies.
\end{abstract}

Index Terms - Boron vacancy, Clustering model, Dielectric breakdown, Hexagonal boron nitride, Ramp voltage stress.

\section{INTRODUCTION}

Two-dimensional (2D) material dielectrics are the key enablers for graphene technology for a wide range of applications including logic, memory, quantum computing, photonics, neuromorphics, sensors etc. Hexagonal boron nitride (h-BN) is a promising $2 \mathrm{D}$ layered insulator with high thermal, mechanical and chemical stability, matching lattice constant to graphene and a van der Waals structure that allows for easy integration and realization of fully-2D nanodevices. There have been a few recent reports on h-BN from a reliability perspective which have: measured the breakdown (BD) field strength [1]; probed the electrical defects using random telegraph noise techniques $[2,3]$; provided physical evidence of the sequential removal of layers during BD with a Weibull distribution to represent BD statistics; observed pit formation in multi-layer h-BN after BD [4]; suggested the presence of bimodality in the progressive BD trends due to competition between lateral wear-out and multi-layer $\mathrm{BD}$ [5]; examined the resonant electron tunneling and electron percolation through local bandgap states within h-BN [6]; and studied the charge trapping and impact ionization phenomena in h-BN at low and high fields [7]. However, there is a lack of insight into the statistical nature and defect chemistry of BD in h-BN [8], which is the focus of this study. We present voltage bias, polarity, temperature and area dependence of the BD phenomenon (and the statistical distribution that best describes it) in chemical vapor deposition (CVD) grown multi-layer hBN films. Additionally, we make use of multi-scale defect-

centric simulations to extract information on the energy level of the precursor defects (traps) that cause BD.

\section{DEVICE FABRICATION}

The sample consists of $\mathrm{h}-\mathrm{BN}$ grown on $\sim 20 \mu \mathrm{m}$ thick polycrystalline $\mathrm{Cu}$ substrate using CVD. The details of h-BN CVD growth have been discussed elsewhere in detail $[9,10]$. Physical analysis using a transmission electron microscope (TEM) shows a variation in thickness of h-BN across the prepared FIB lamella ranging between $\sim 2-4$ nm (i.e. $\sim 5-12$ layers of h-BN) as shown in Figs. 1(a)-(d). An EELS spectral line is acquired to confirm the elemental composition across the layers as shown in Fig. 1(e). As expected from the CVD growth process of h-BN, residual Boron and Nitrogen atoms can be observed in the $\mathrm{Cu}$ substrate as well.
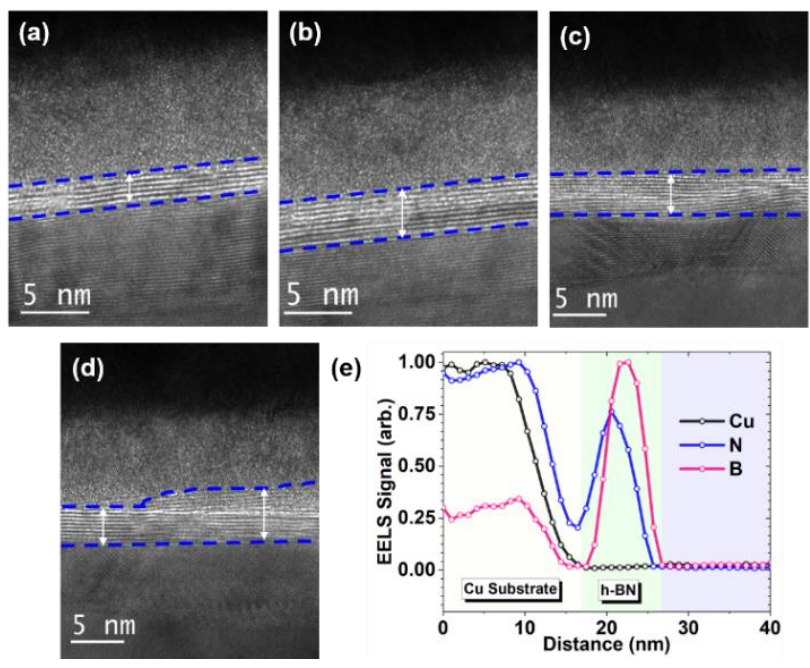

Fig. 1. TEM micrographs showing multi-layer h-BN grown on $\mathrm{Cu}$ substrate showing (a) $\sim 5-6$ layers (i.e. $t_{h-B N} \sim 2 \mathrm{~nm}$ ) and (b, c) $\sim 10-12$ layers (i.e. $t_{h-B N}$ $\sim 4 \mathrm{~nm}$ ) at different regions of the film. (d) Overlap of h-BN layers across two grains during CVD growth. Note that all the TEM micrographs shown correspond to different regions of the same FIB lamella of length $\sim 5 \mu \mathrm{m}$ prepared for TEM analysis. (e) Electron energy loss spectra (EELS) showing the elemental distribution of Boron, Nitrogen and Copper.

Top electrodes have been defined on h-BN/Cu using a shadow mask with $\mathrm{Cr}(\sim 7 \mathrm{~nm})$ and $\mathrm{Au}(\sim 50 \mathrm{~nm})$ deposition using ebeam evaporation to form $\mathrm{Au} / \mathrm{Cr} / \mathrm{h}-\mathrm{BN} / \mathrm{Cu}$ capacitors. The area of the top electrode for the test capacitors is $25 \times 25 \mu \mathrm{m}^{2}$. 
During electrical measurements, a bias is applied to the $\mathrm{Cr} / \mathrm{Au}$ while the $\mathrm{Cu}$ substrate is electrically grounded. For the conduction atomic force microscope (CAFM) measurements (done in ultra-high vacuum with a base pressure of $2 \times 10^{-10}$ Torr), we have used blanket films $(\mathrm{h}-\mathrm{BN} / \mathrm{Cu})$ where bias is applied to the CAFM tip while the substrate is grounded, akin to device level measurements. For the measurements done on capacitor devices and on blanket films using CAFM, breakdown voltage $\left(V_{B D}\right)$ is defined as the voltage at which current reaches $\sim 1 \mathrm{~mA}$ and $\sim 1 \mathrm{nA}$, respectively.

\section{RESULTS AND DISCUSSION}

\section{A. Constant Voltage Stress Tests}

To examine the time-dependent leakage evolution trends in h$\mathrm{BN}$, constant voltage stress (CVS) TDDB tests were conducted at $0.30-0.70 \mathrm{~V}$ for at least $\sim 10$ capacitor devices, as shown in Fig. 2. We observe a relatively flat phase of leakage in the stress induced leakage current (SILC) regime followed by a gradual increase in current and defect induced noise with time, ending up in multiple step wise BD trends (logarithmic jumps), possibly due to sequential layer-by-layer $\mathrm{BD}$, as advocated by Hattori et al. [4]. Precise control over the discrete steps of BD appears to be difficult to achieve.

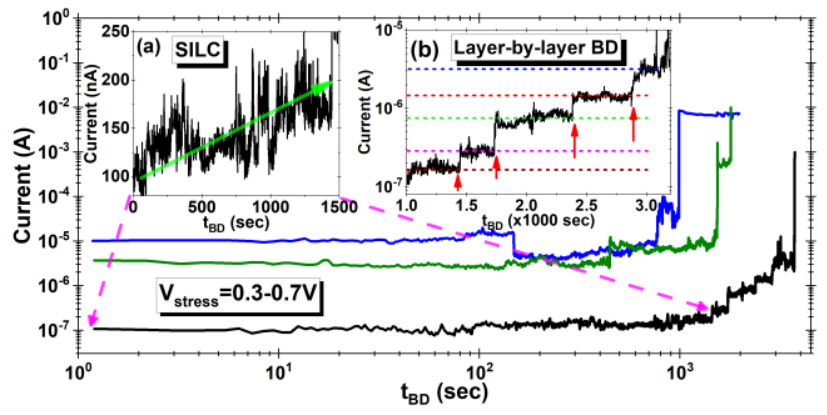

Fig. 2. Leakage current evolution during CVS test on multiple h-BN capacitors showing linear (zoom-in in (a)) and logarithmic jumps (zoom-in in (b)) corresponding to random vacancy generation and layer-by-layer stepwise $\mathrm{BD}$, respectively. Logarithmic jumps are consistently seen in the later stages.

\section{B. Ramp Voltage Stress Tests}

Considering inherent variations in thickness of CVD grown multi-layer h-BN, we use ramped voltage stress (RVS) measurements at device level for time-efficient BD study. The $V_{B D}$ data were plotted for three different ramp rates in Fig. 3(a) on a Weibull plot. At least $\sim 35$ devices have been stressed for each ramp rate. A clear positive correlation of $V_{B D}$ on ramp rate was observed, akin to $\mathrm{HfO}_{2} / \mathrm{SiO}_{2}$, implying that the "extrinsic" thickness fluctuation effects are equally embedded in all the devices tested and that the trends observed are "intrinsic" to the degradation kinetics of h-BN.

A careful analysis of $V_{B D}$ data shows poor fitting to a Weibull distribution at high percentiles, as shown in Fig. 3(b). Using the defect clustering model (DCM) (Eqn. 1), proposed by Wu et al. $[\mathbf{1 1}, \mathbf{1 2}]$, we fit the data set of the smallest ramp rate using both standard Weibull model and the DCM (in which, the additional parameter, $\alpha_{c}$ is the cluster factor and a lower $\alpha_{c}$ implies more defect clustering, while $\beta$ is the Weibull slope).
We find a lower Akaike Information Criterion (AIC) value for DCM suggesting it is the better representation of the data set $[13,14]$. We postulate that defect clustering can originate due to (a) defect generation near pre-existing defect sites with lower activation energy and higher binding energy for multivacancy configurations, (b) process-induced thickness variations (as shown in Fig. 1), (c) grain boundaries in polycrystalline $\mathrm{h}$-BN serving as thermodynamic sink for migration of $\mathrm{B}$ vacancies and/or (d) non-uniform field distributions across the CAFM tip. The predominant casual factor of the clustering behavior here requires more in-depth study to be carried out in the future.

$$
F=1-\left(1+\frac{1}{\alpha_{c}} \cdot\left(\frac{V_{B D}}{V_{B D-63 \%}}\right)^{\beta}\right)^{-\alpha_{C}}
$$
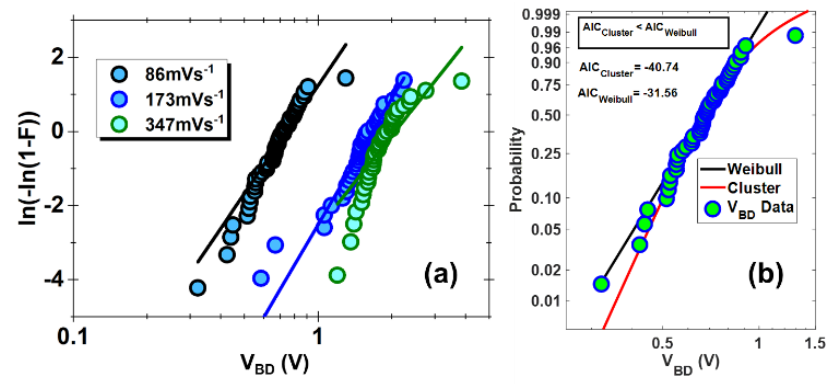

Fig. 3. (a) Weibull plot of device-level $V_{B D}$ data for three different ramp rates along with Weibull fittings. (b) Weibull and cluster model fits to smallest ramp rate RVS $V_{B D}$ data in (a) with AIC values indicated. Lower AIC value implies a better fit.

\section{Investigation of Area Scaling}

To examine area scaling, we present the $V_{B D}$ data extracted from CAFM (estimating the effective area probed by this tip to be $\sim 100 \times 100 \mathrm{~nm}^{2}$ ) and device level measurements in Fig. 4(a). With a difference in physical area of more than three orders of magnitude, the application of the area scaling law still shows good agreement. This suggests that defect generation in h-BN is "random" in space to begin with, before the positive feedback effects kick in to localize the BD percolation path. Moreover, the number of preferential BD spots (weakest links) is expected to scale with area as well, assisted by the polycrystallinity of h-BN with random grain size distribution.
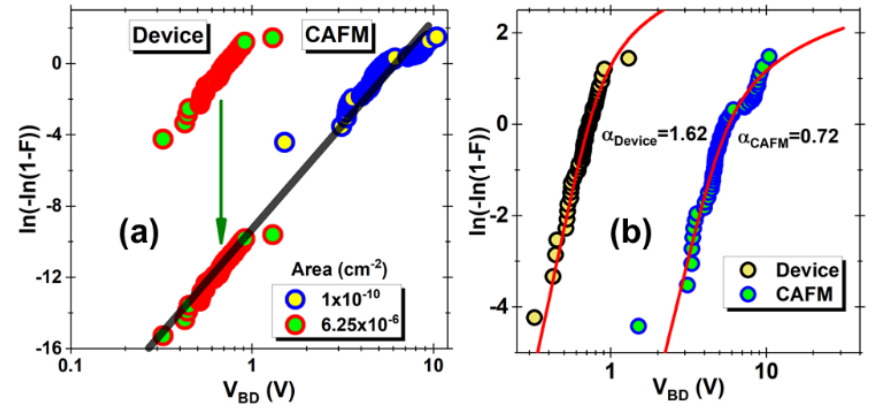

Fig. 4. (a) Strong validity of Poisson area scaling of BD comparing CAFM and device level BD data. (b) Cluster model fitting to both CAFM $\left(\alpha_{C A F M}=\right.$ $0.72)$ and device-level $\left(\alpha_{\text {Device }}=1.62\right) V_{B D}$ data.

Comparing the cluster model fit for device and CAFM BD data in Fig. 4(b), we observe lower $\alpha_{c}$ for CAFM data possibly 
due to locally amplified h-BN film thickness variations [15] and non-homogeneous field from the CAFM tip. The value of $\alpha_{c}$ was not constrained to be the same for CAFM and device level tests as the extent of clustering could be different, most likely due to the measurement of a large variety of grain/grain boundary and differing thickness regions in the large area devices.

\section{Polarity Dependence of Breakdown}

With the intention to identify the driving forces for h-BN BD, polarity dependent $I-V$ data were collected at device level (Fig. 5(a)) and corresponding $V_{B D}$ data plotted in Fig. 5(b), with a very different ramp rate for $+V_{\text {stress }}$ and $-V_{\text {stress. }}$. While the Weibull slope $(\beta)$ remains unchanged (we assume Weibull for simplicity here), the leakage current and $V_{B D}$ are highly polarity dependent. Better immunity to $\mathrm{BD}$ is observed for negative bias applied to the top electrode, corresponding to lower leakage current (black traces in Fig. 5(a)). This suggests that defect generation is not purely field-driven and carrier fluence / charge trapping play a critical role in assisting BD of h-BN.
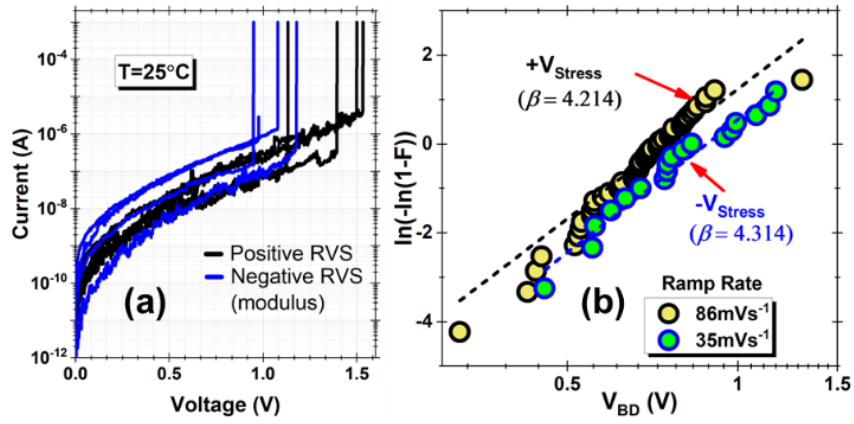

Fig. 5. (a) Device-level $I-V$ plot of BD trends in the positive and negative polarity stress modes showing asymmetry in the tunneling current. (b) Weibull plot of $V_{B D}$ with positive and negative bias polarities at very different ramp rates.

\section{E. Extracting the Defect Energetics in h-BN}

We perform temperature-dependent $\mathrm{BD}$ studies on h-BN devices $\left(\mathrm{T}=25^{\circ} \mathrm{C}, 50^{\circ} \mathrm{C}\right)$ (Fig. $\left.6(\mathrm{a}, \mathrm{b})\right)$ and use $I-V$ data sets to probe the energetics of the defect using simulations from Ginestra $^{\mathrm{TM}}$ software, a multi-scale defect-centric simulation platform that self-consistently describes all degradation mechanisms for dielectrics and 2D materials. Charge transport calculations are performed considering local potential (given by applied bias and defect charge state and occupancy) while accounting for several conduction mechanisms: direct/FowlerNordheim tunneling, thermionic emission, drift, diffusion, and phonon trap-assisted-tunneling, the latter being dominant in high-א.

The simulation results, shown in Fig. 6(c, d), nicely reproduce the measured $I-V$ data using a single set of model parameters, only when the dependence of the h-BN band-gap on temperature is properly considered $(\sim 10 \mathrm{meV} / \mathrm{K}$, close to the value reported in [16] for h-BN nanosheets). This high sensitivity of bandgap to temperature is unique to h-BN and is not critical when considering charge transport in bulk high-к. Model fitting to the data shows that charge transport is assisted by defects located between $\sim 2-3 \mathrm{eV}$ from the bottom of the conduction band of h-BN with a relaxation energy $\left(E_{R E L}\right)$ of $\sim 1.5 \mathrm{eV}$. These extracted values are consistent with those reported for B-vacancies and complex anti-sites $[\mathbf{1 7}, \mathbf{1 8}]$.

The $V_{B D}$ values at $63^{\text {rd }}$ percentile in Fig. $6(\mathrm{~b})$ for $\mathrm{T}=\left\{25^{\circ}\right.$, $\left.50^{\circ}\right\} \mathrm{C}$ were used to estimate effective $\mathrm{B}-\mathrm{N}$ bond breakage activation energy, $E_{A}$ (using thermochemical formalism [19]), assuming that the corresponding bond-breaking rates are equal at $\mathrm{BD}$, as described in Ref. [20, 21]. The value of $E_{A} \sim 1.6 \mathrm{eV}$, obtained by assuming a bond polarization factor $(b)$ of $\sim 10 \mathrm{e} \AA$, perfectly agrees with to describe defect generation processes involved in resistive switching phenomenon in h-BN [22], and effectively incorporates possible effects of carrier fluence and charge trapping.
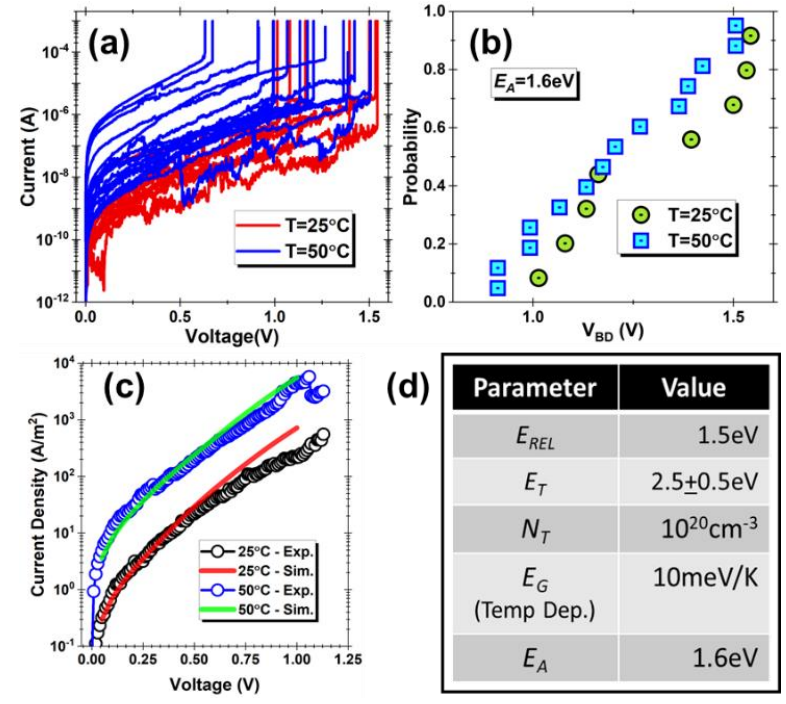

(d)

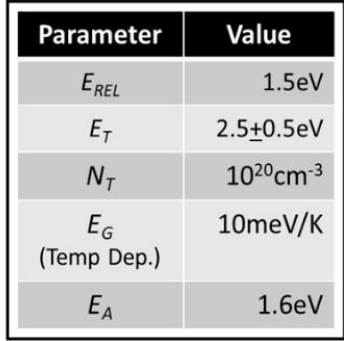

Fig. 6. (a) $I-V$ plot of $\mathrm{h}-\mathrm{BN}$ devices at $25^{\circ} \mathrm{C}$ and $50^{\circ} \mathrm{C}$, showing a logarithmic increase in leakage current profiles with temperature. (b) Weibull plot of $V_{B D}$ data at $25^{\circ} \mathrm{C}$ and $50^{\circ} \mathrm{C}$. (c) Fitting of multi-phonon trap-assisted tunneling (TAT) charge transport model to the average measured current density in hBN devices. The averaged leakage profile for $25^{\circ} \mathrm{C}$ and $50^{\circ} \mathrm{C}$ in (a) is extracted for model fitting here. (d) Extracted values of the trap parameters in h-BN including defect density $\left(N_{T}\right)$, relaxation and thermal ionization energy $\left(E_{R E L}, E_{T}\right)$, band gap $\left(E_{G}\right)$ temperature coefficient and zero-field activation energy $\left(E_{A}\right)$.

\section{CONCLUSION}

We have used a suite of ramp voltage measurements at different ramp rate conditions on large capacitor devices and blanket h-BN films (using CAFM) to study the statistical nature of dielectric breakdown (BD) in these films. The energetics of the defects that serve as precursors for BD were identified by charge transport simulation fits to our $I-V$ data. In the 2-4nm CVD grown h-BN films studied here, BD was found to be polarity dependent and area scaling was found to be valid. In addition, defect clustering was shown to be prominent likely due to thickness variations and microstructural inhomogeneity (grain boundaries). We have identified boron vacancies as the critical defect type that assists the overall charge transport and BD in h-BN films. 


\section{REFERENCES}

[1] L. Britnell, R. V. Gorbachev, R. Jalil, B. D. Belle, F. Schedin, M. I. Katsnelson, L. Eaves, S.V. Morozov, A.S. Mayorov, N.M.R. Peres, A.H.C. Neto, J. Leist, A.K. Geim, L.A. Ponomarenko and K.S. Novoselov, "Electron tunneling through ultrathin boron nitride crystalline barriers", Nano Letters, Vol. 12, Issue 3, pp. 1707-1710, (2012). DOI: $10.1021 / \mathrm{nl} 3002205$

[2] A. Ranjan, F. M. Puglisi, N. Raghavan, S. J. O'Shea, K. Shubhakar, P. Pavan, A. Padovani, L. Larcher and K.L. Pey, "Random telegraph noise in 2D hexagonal boron nitride dielectric films", Applied Physics Letters, Vol. 112, Issue 13, 133505, (2018). DOI: 10.1063/1.5022040

[3] A. Ranjan, N. Raghavan, S.J. O'Shea, S. Mei, M. Bosman, K. Shubhakar and K.L. Pey, "Conductive atomic force microscope study of bipolar and threshold resistive switching in 2D hexagonal boron nitride films", Scientific Reports, Vol. 8, Issue 1, 2854, (2018). DOI: 10.1038/s41598-018-21138-x

[4] Y. Hattori, T. Taniguchi, K. Watanabe, and K. Nagashio, "Layer-bylayer dielectric breakdown of hexagonal boron nitride", ACS Nano, Vol. 9, Issue 1, pp. 916-921, (2015). DOI: 10.1021/nn506645q

[5] Y. F. Ji, C. B. Pan, M. Y. Zhang, S. B. Long, X. J. Lian, F. Miao, F. Hui, Y. Shi, L. Larcher, E. Wu and M. Lanza, "Boron nitride as twodimensional dielectric: Reliability and dielectric breakdown", Applied Physics Letters, Vol. 108, Issue 1, 012905, (2016). DOI: $10.1063 / 1.4939131$

[6] M. T. Greenaway, E. E. Vdovin, D. Ghazaryan, A. Misra, A. Mishchenko, Y. Cao, Z, Wang, J.R. Wallbank, M. Holwill, Y.N. Khanin, S.V. Morozov, K. Watanabe, T. Taniguchi, O. Makarovsky, T.M. Fromhold, A. Patane, A.K. Geim, V.I. Falko, K.S. Novoselov and L. Eaves, "Tunnel spectroscopy of localised electronic states in hexagonal boron nitride", Communications Physics, Vol. 1, Issue 1, (2018). DOI: 10.1038/s42005-018-0097-1

[7] Y. Hattori, T. Taniguchi, K. Watanabe, and K. Nagashio, "Impact ionization and transport properties of hexagonal boron nitride in a constant-voltage measurement", Physical Review B, Vol. 97, Issue 4, 045425, (2018). 10.1103/PhysRevB.97.045425

[8] K.L. Pey, A. Ranjan, N. Raghavan, K. Shubhakar and S.J. O'Shea, "Dielectric Breakdown in 2D Layered Hexagonal Boron Nitride - The Knowns and the Unknowns", 2019 IEEE International Reliability Physics Symposium (IRPS), Monterey, California, USA, pp. 4E.1-14E.1-12, (2019), DOI: 10.1109/IRPS.2019.8720405.

[9] Y. Shi, C. Hamsen, X. Jia, K. K. Kim, A. Reina, M. Hofmann, A.L. Hsu, K. Zhang, H. Li, Z.Y. Juang, M.S. Dresselhaus, L.J. Li and J. Kong "Synthesis of few-layer hexagonal boron nitride thin film by chemical vapor deposition", Nano Letters, Vol. 10, Issue 10, (2010). DOI: $10.1021 / \mathrm{nl} 1023707$

[10] J. S. Lee, S. H. Choi, S. J. Yun, Y. I. Kim, S. Boandoh, J.-H. Park, B.G. Shin, H. Ko, S.H. Lee, Y.M. Kim, Y.H. Lee, K.K. Kim and S.M. Kim, "Wafer-scale single-crystal hexagonal boron nitride film via selfcollimated grain formation," Science, Vol. 362, Issue 6416, pp. 817-821, 2018. DOI: $10.1126 /$ science.aau2132

[11] E. Wu, B. Li and J.H. Stathis, "Modeling of time-dependent nonuniform dielectric breakdown using a clustering statistical approach", Applied Physics Letters, Vol. 103, Issue 15, 152907, (2013). DOI: $10.1063 / 1.4824035$

[12] E. Wu, B. Li, J.H. Stathis and C. LaRow, "Time-dependent clustering model versus combination-based approach for BEOL/MOL and FEOL non-uniform dielectric breakdown: Similarities and disparities", 2014 IEEE International Reliability Physics Symposium (IRPS), pp. 5B.2-15.B.2-7, (2014). DOI: 10.1109/IRPS.2014.6860662

[13] H. Akaike, "A new look at the statistical model identification," IEEE Transactions on Automatic Control, Vol. 19, Issue 6, pp. 716-723, (1974). DOI: 10.1109/TAC.1974.1100705

[14] T.Bučar, M. Nagode and M.Fajdiga, "Reliability approximation using finite Weibull mixture distributions", Reliability Engineering \& System
Safety, Vol. 84, Issue 3, pp. 241-251, (2004). DOI: 10.1016/j.ress.2003.11.008

[15] A. Ranjan, N. Raghavan, S. J. O'Shea, S. Mei, M. Bosman, K. Shubhakar and K.L. Pey, "Mechanism of soft and hard breakdown in hexagonal boron nitride 2D dielectrics", 2018 IEEE International Reliability Physics Symposium (IRPS), Burlingame, California, USA, pp. 4A.1-1-4A.1-6, (2018). DOI: 10.1109/IRPS.2018.8353574

[16] P. Kumbhakar, A.K. Kole, C.S. Tiwary, S. Biswas, S. Vinod, J.T. Tijerina, U. Chatterjee and P.M. Ajayan, "Nonlinear optical properties and temperature-dependent uv-vis absorption and photoluminescence emission in $2 \mathrm{~d}$ hexagonal boron nitride nanosheets", Advance Optical Materials, Vol. 3, Issue 6, pp. 828-835, (2015). DOI: 10.1002/adom.201400445

[17] M. Abdi, J.P. Chou, A. Gali and M.B. Plenio, "Color centers in hexagonal boron nitride monolayers: a group theory and ab initio analysis", ACS Photonics, Vol. 5, Issue 5, pp. 1967-1976, (2018). DOI: 10.1021/acsphotonics.7b01442

[18] W. Orellana and H. Chacham, "Atomic geometry and energetics of native defects in cubic boron nitride", Brazilian Journal of Physics, Vol. 29, Issue 4, pp. 801-805, (1999). DOI: 10.1590/S010397331999000400040

[19] J. McPherson, J.Y. Kim, A. Shanware and H. Mogul, "Thermochemical description of dielectric breakdown in high dielectric constant materials", Applied Physics Letters, Vol. 82, Issue 13, 2121, (2003). DOI: $10.1063 / 1.1565180$

[20] M. Alayan, E. Vianello, A. Padovani, P. Blaise, B. Sklénard, C. Charpin, S. Bernasconi, A. Roule, C. Cagli, E. Jalaguier, B. De Salvo, L. Larcher, L. Perniola, "Different $\mathrm{Al}$ incorporation methods in $\mathrm{HfO}_{2}$ based RRAM and their effects on forming and thermal stability", 19th Workshop on Dielectrics in Microelectronics, Italy, (2016).

[21] M. Alayan, E. Vianello, B.D. Salvo, L. Perniola, A. Padovani and L. Larcher, "Correlated Effects on Forming and Retention of Al Doping in HfO2-Based RRAM", IEEE Design \& Test, Vol. 34, Issue 3, pp. 23-30, (2017). DOI: 10.1109/MDAT.2017.2682246

[22] F. M. Puglisi, L. Larcher, C. Pan, N. Xiao, Y. Shi, F. Hui and M. Lanza, "2D h-BN based RRAM devices", 2016 IEEE International Electron Devices Meeting (IEDM), (2016). DOI: 10.1109/IEDM.2016.7838544 